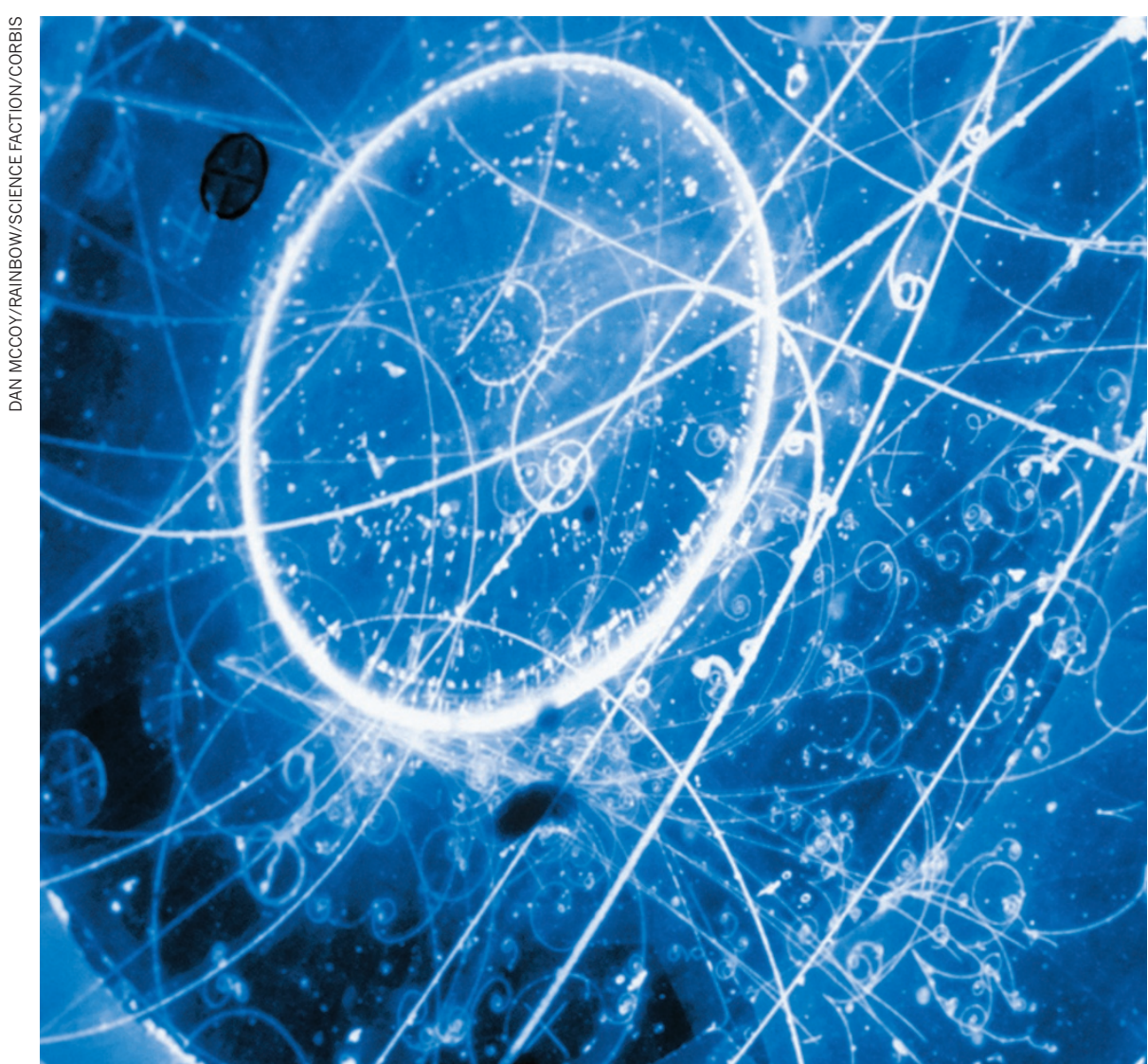

Neutrinos are among the many types of particles produced when electrons and positrons collide.

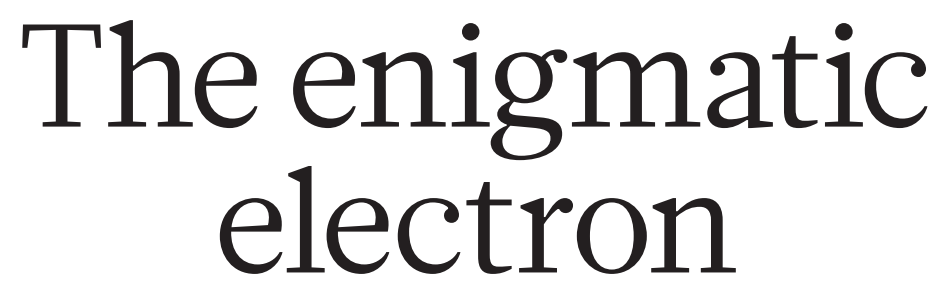

While we are still learning about the particle's true nature, says Frank Wilczek, let's celebrate its beauty.

W hat is an electron? That question was central to the development of quantum theory early in the twentieth century, and remains at the frontier of physics today. There are several inconsistent answers, each correct. A century after Danish physicist Niels Bohr conceived of the electron as the proton's satellite ${ }^{1}$, our perception of the electron continues to evolve and expand.

Bohr's answer to this question in 1927 epitomized his beloved concept of complementarity: in some circumstances electrons are best described as particles, with definite positions; in others as waves, with definite momenta ${ }^{2}$. Both descriptions are valid and useful, yet according to Heisenberg's uncertainty principle, they are mutually exclusive: positions and momenta cannot be known accurately at the same time. Each depiction captures an aspect of the electron's nature, but neither exhausts it.

Modern quantum theory reinforces Bohr's conclusion that what you see depends on how you choose to look. Electrons are both ideally simple and unimaginably complex. They are understood with precision yet remain mysterious. Electrons are stable bedrock in physicists' world picture, and are playthings that we are learning to fragment and transform.

\section{SIMPLE AND COMPLEX}

For most practical purposes, an electron is a structureless particle with an intrinsic angular momentum, or spin. Just two

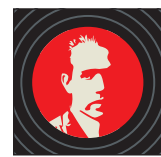

THE QUANTUM ATOM A Nature special issue nature.com/bohr100 numbers - the electron's mass and its electric charge - fuel the equations that describe its behaviour. From this 'practical electron' model, physicists constructed modern microelectronics. It is also the working foundation for chemistry, including biochemistry.

But to a high-energy positron (antielectron), an electron is a cornucopia. Collisions of electrons and positrons, such as those carried out at the Large ElectronPositron (LEP) collider at CERN, Europe's particle-physics lab near Geneva in Switzerland, produce streams of quarks, gluons, muons, tau leptons, photons and neutrinos. To understand the complexity of an electron, all of the esoteric resources of modern physics must be brought to bear.

There is tension between these two observations, that the electron is a simple pointparticle, and that it contains the world. They can be reconciled through a concept that I call quantum censorship, whereby properties of objects vary according to the energy with which they are probed. Quantum censorship was implicit in Bohr's atomic model and, in a more general form, remains a central pillar of modern quantum theory.

In his 1913 model of the hydrogen atom ${ }^{1}$, Bohr pictured an electron orbiting the proton like a planet in a miniature Solar System. As he knew, and the physicist James Clerk Maxwell had emphasized before him, mechanical models of the atom have severe problems. They predict a variety of hydrogen atoms, with different orbital shapes and sizes, whereas in reality, all hydrogen atoms are identical. The models also predict that atoms are unstable, because moving electrons should radiate energy and spiral into the central proton, which clearly they do not.

Bohr boldly assumed away those difficulties. He restricted electrons to a set of discrete, or quantized, energy states within an atom to avoid instability. He recognized that the level with the lowest energy, or ground state, has a finite size, keeping the electron and proton apart.

Today, we trace Bohr's rules to the fact that the proper quantum-mechanical description of electrons involves wave functions, the oscillation patterns of which are standing waves. The equations that govern electrons in atoms are similar to those for vibrations in musical instruments, which produce scales of distinct tones.

The same ideas apply to complex, bound systems, such as atoms that have many electrons and larger nuclei. A system in its ground state tends to remain there, if little energy is fed in, betraying no evidence of its internal structure. Only when it is excited into a higher state do complexities emerge. This is the essence of quantum censorship. Thus, below an energy threshold, atoms 
appear to be the "hard, massy, impenetrable" units that Isaac Newton inferred. Above it, their components can be torn out.

Similarly, electrons, despite the fecundity that they showed at the LEP collider, betray nothing of their inner workings at low energies. An electron's structure is revealed only when one supplies enough energy to unleash electron-positron pairs - at least 1 megaelectronvolt, which corresponds to the unearthly temperature of $10^{10}$ kelvin. Thus the practical electron is not an approximation to reality, in the usual sense of fuzziness; rather, it is a precise description that applies under limited (albeit quite generous) conditions.

Having recognized its power, let us celebrate the practical electron's intellectual beauty. Each of its properties is intimately connected to profound symmetries of physical law. Mass and spin classify all possible realizations of special relativity by particles. Electric charge, a conserved quantity, classifies realizations of the 'gauge symmetry' of electromagnetism. Specifying how the practical electron responds to those symmetry transformations determines its physical behaviour. The electron is thus an embodiment of symmetry: its physical properties are inherent to its mathematical form.

\section{PRECISE AND MYSTERIOUS}

In principle, electrons can possess both magnetic- and electric-dipole fields, the axes of which are set by the electron's spin. But the status of these fields could hardly be more different. The strength of the electron's magnetic field provides perhaps the most stringent and brilliantly successful comparison of theory and experiment in all of physical science, whereas the value of the electric field has never been measured. It is a mystery even to theory.

Establishing the strength of the electron's magnetic field - in terms of a gyromagnetic ratio or ' $g$-factor' - was a major focus of twentieth-century physics. An early triumph of physicist Paul Dirac's 1928 relativistic wave equation for the electron ${ }^{3}$ was its suggestion that $g=2$, which was found to be nicely consistent with atomic spectroscopy.

Post-war developments in precision spectroscopy, using atomic beams, revealed that $g$ deviated from that value by one part in 1,000 . Theorists matched that deviation when they had mastered the mathematical difficulties of quantum-field theory enough to calculate corrections to the Dirac equation to account for quantum fluctuations (the energy of which release virtual photons).

Creative dialogue between experiment and theory continues today, with improved accuracy on each side allowing ever more rigorous comparisons. The experimental frontier has moved to beautiful investigations of single electrons in electric and magnetic traps.
Theoretical calculations have become intricate, now including fluctuations in fluctuations in fluctuations. The value of $g$ is known to a dozen significant digits ${ }^{4}$.

A crude but appealing 'explanation' of the origin of the electron's magnetic field is that quantum uncertainty in position smears the electron's charge over a volume, which rotates because of the electron's spin. The electron is effectively a spinning ball of charge, and elementary electromagnetism "Anelectron's erates a magneticstructure dipole field. The size is revealed of that ball can be estionlywhen mated to be roughly one supplies $2.4 \times 10^{-12}$ metres. enough Attempts to pin down energy." an electron's position more accurately than this require, according to the uncertainty principle, injecting the electron with so much energy that extra electrons and antielectrons are produced, confusing the identity of the original electron.

An electric dipole, should it exist, would generate broadly similar corrections. But no such field has been detected. Great efforts have gone into the experimental search, using all the tricks and traps that revealed the magnetic moment. So far there is only an upper bound for the electric dipole moment ${ }^{5}$. This is an extraordinary 17 orders of magnitude smaller than one might expect - naively, given the electron's effective size.

Why is it so hard for spin to align electric charge? One explanation involves time-reversal symmetry. If we run time backwards, the laws of physics stay the same. But for a spinning electron, the north and south poles would swap. Thus an electric dipole accumulating charge at one pole violates time-reversal symmetry.

But nature does not always respect timereversal symmetry, as we know from observations of $K$ and $B$ mesons $^{6}$. So a non-zero electric dipole moment for electrons is a theoretical possibility. It is tantalizing that values of the electric field that lie just below the present upper bound are expected in many theories of physics beyond the standard model of particle physics, including supersymmetry. Ingenious experiments using solid-state physics and molecular spectroscopy have been proposed in a bid to search more sensitively for the existence of tiny electric fields that are generated by reorienting spins. This 'other' dipole moment might prove to be a focus for twenty-firstcentury physics.

\section{RIGID AND PROTEAN}

Electrons are rigid and defend their integrity stoutly. They follow the Pauli exclusion principle, which states that no two electrons can be in the same quantum state at the same time. This is the defining characteristic of fermions, a class of particles that includes protons, neutrons and electrons. As a result, electrons cannot be crushed.

Nature's most imposing macroelectronic creation is the white-dwarf star. The Sun will become such a star 4 billion to 5 billion years from now, when it has exhausted its nuclear fuel, causing it to collapse into a sphere roughly the size of Earth, but a million times more dense. White dwarfs rely on the quantum statistics of electrons for their support. Squeezing electrons together promotes some into higher energy states, exerting a force or 'degeneracy pressure' that balances gravity and halts further collapse.

But subtle collective action can achieve what raw pressure does not, and fragment electrons. This has been extensively studied in electron states in thin semiconductor interfaces that are extremely pure and cold, and are subjected to strong magnetic fields. These states are known as fractional quantum Hall effect liquids ${ }^{7}$. Their electric currents reveal the presence of particles whose charge is a fraction of an electron's.

Electrons also lose their individual identities in superconductors, in which electrons pair up to form a pervasive sea. Thus, electrons become their own antiparticles. By combining fragmentation with superconductivity, we can get half-electrons that are their own antiparticles. Such 'Majorana modes' have now been observed experimentally ${ }^{8}$ and promise to have exotic properties. Notably, their quantum state retains 'memories' of how they were created and where they have been. Manipulating electron fragments opens up rich new possibilities for microelectronics and quantum computing, which are only beginning to be explored.

So, what is an electron? An electron is a particle and a wave; it is ideally simple and unimaginably complex; it is precisely understood and utterly mysterious; it is rigid and subject to creative disassembly. No single answer does justice to reality.

Frank Wilczek is professor of physics at the Center for Theoretical Physics, Massachusetts Institute of Technology, Cambridge, Massachusetts 02139, USA. e-mail:wilczek@mit.edu

1. Bohr, N. Philos. Mag. 26, 1-25 (1913).

2. Bohr, N. Nature 121, 580-590 (1928)

3. Dirac, P. A. M. Proc. R. Soc. A 117, 610-624 (1928).

4. Mohr, P. J., Taylor, B. N. \& Newell, D. B. Rev. Mod. Phys. 84, 1527-1605 (2012).

5. Hudson, J. J. et al. Nature 473, 493-496 (2011).

6. Kirkby, D. \& Nir, Y. 'CP violation in meson decays' in Beringer, J. et al. (Particle Data Group) Phys. Rev. D 86, 010001 (2012).

7. Das Sarma, S., Pinczuk, A. (eds) Perspectives in Quantum Hall Effects (Wiley-VCH, 1996).

8. Mourik, V. et al. Science 336, 1003-1007 (2012). 